SHORT TITLE: $\quad$ Reading, self-esteem, and child mental health

Relationships between reading ability and child mental health: Moderating effects of self-esteem

Mark E. Boyes ${ }^{a}$, Bree Tebbutt $^{b}$, Kathryn A. Preece $^{c}, \&$ Nicholas A. Badcock ${ }^{c}$

a: School of Psychology and Speech Pathology, Faculty of Health Sciences, Curtin University, Perth, Australia

b: School of Psychology, University of Newcastle, Newcastle, Australia

c: ARC Centre of Excellence in Cognition and its Disorders, Department of Cognitive Science, Macquarie University, Sydney, Australia

\title{
Citation:
}

Boyes, M. E., Tebbutt, B., Preece, K., \& Badcock, N. A. (in press). Relationships between reading ability and child mental health: Moderating effects of self-esteem. Australian Psychologist. DOI: 10.1111/ap.12281.

NOTICE: this is the author's version of a work that was accepted for publication in the Australian Psychologist. Changes resulting from the publishing process, such as peer review, editing, corrections, structural formatting, and other quality control mechanisms may not be reflected in this document. Changes may have been made to this work since it was submitted for publication. A definitive version was subsequently published in the Australian Psychologist, DOI: 10.1111/ap.12281. 
Reading, self-esteem, and child mental health 2

\begin{abstract}
Objective: Children with reading difficulties are at elevated risk for externalising (e.g. conduct disorder) and internalising (e.g. anxiety and depression) mental health problems. Reading ability is also negatively associated with self-esteem, a consistent predictor of child and adolescent mental health more broadly. This study examined whether self-esteem moderated and/or mediated relationships between reading ability and mental health. Method: 117 children (7-12 years) completed standardised reading assessments (Castles and Coltheart Test 2) and self-report measures of mental health (Strengths and Difficulties Questionnaire) and self-esteem (Coopersmith Self-esteem Inventory). Non-verbal IQ was measured using the block design and matrix reasoning subscales of the Wechsler Abbreviated Scale of Intelligence, and was controlled for in all multivariate analyses. Results: Reading ability was negatively associated with internalising symptoms. This relationship was not moderated by self-esteem. Poor readers also reported more total difficulties and externalising symptoms, but only at low-levels of self-esteem. There was no evidence that self-esteem mediated relationships between reading ability and mental health. Conclusions: Poor reading was associated with internalising symptoms. Selfesteem moderated the impact of reading ability on total difficulties and externalising symptoms, with high self-esteem buffering against negative impacts of poor reading. However, the reliability of the self-esteem scale used in the study was poor and findings need replication using a reliable and valid self-esteem measure, as well as other measures of child mental health. If replicated, future research should examine whether interventions aiming to improve self-esteem can reduce the risk of externalising problems in children with reading difficulties.
\end{abstract}

Keywords: reading, mental health, externalising, internalising, self-esteem, children 
Reading, self-esteem, and child mental health 3 Approximately 5-10\% of children have substantial difficulties in learning to read (Boetsch, Green, \& Pennington, 1996; Maughan \& Carroll, 2006), representing between two and four children in an average classroom. Reading ability is associated with mental health problems (Carroll, Maughan, Goodman, \& Meltzer, 2005; Maughan \& Carroll, 2006; Willcutt \& Pennington, 2000b), with poor readers at elevated risk for both externalising problems (disruptive behaviours, such as conduct problems and hyperactivity) and internalising problems (emotional symptoms, such as depression and anxiety). However, little research has examined why reading ability is associated with these negative outcomes. If we are to design effective interventions to promote mental health amongst children with reading difficulties, it is essential to identify potentially malleable mechanisms explaining psychological outcomes associated with reading ability, which can then become targets of future intervention efforts (Boyes, Leitao, Claessen, Badcock, \& Nayton, in press). The current study aimed to investigate the possibility that the relationship between reading ability and child mental health may be moderated and/or mediated by self-esteem.

\section{Reading and child mental health}

A growing body of literature has established links between children's reading ability and both externalising and internalising symptoms (Carroll et al., 2005; Maughan \& Carroll, 2006; Willcutt \& Pennington, 2000b). The majority of this research has focused on externalising problems (Hinshaw, 1992; Maughan \& Carroll, 2006), and given that these disruptive behavioural symptoms are observable and create classroom difficulties for teachers to manage, this is perhaps unsurprising. Reading difficulties are highly comorbid with attentiondeficit/hyperactivity disorder (ADHD; Willcutt \& Pennington, 2000a), with as many as 15-40\% of children with reading difficulties also meeting criteria for ADHD (Maughan \& Carroll, 2006; Willcutt \& Pennington, 2000a). However, the relationship between reading ability and ADHD appears to be specific to attentional problems with less evidence of a link between reading ability 
Reading, self-esteem, and child mental health 4 and hyperactivity (Carroll et al., 2005). Reading difficulties have also been associated with other disruptive behavioural disorders. Early epidemiological studies noted that poor readers were at substantially elevated risk of anti-social behaviour and conduct disorder (Rutter \& Yule, 1970) and there is now a substantial body of research linking reading difficulties with conduct disorder, delinquency, aggression, and oppositional defiant disorder (Arnold et al., 2005; Carroll et al., 2005; Frick et al., 1991; Willcutt \& Pennington, 2000b). However, due to the comorbidity between reading problems and ADHD some of these relationships may be epiphenomenal (Maughan \& Carroll, 2006). Indeed, a number of studies have demonstrated that the relationships between reading ability and externalising problems may be mediated by ADHD (Rietz, Hasselhorn, \& Labuhn, 2012; Willcutt \& Pennington, 2000b) and, more specifically, inattention (Arnold et al., 2005; Carroll et al., 2005).

Less research has examined relationships between reading ability and internalising symptoms or emotional problems (Maughan \& Carroll, 2006), although reading difficulties have been associated with depression (Arnold et al., 2005), anxiety (Arnold et al., 2005; Carroll et al., 2005; Whitehouse, Spector, \& Cherkas, 2009), somatic complaints (Arnold et al., 2005), low mood (Carroll et al., 2005), and general socio-emotional problems (Terras, Thompson, \& Minnis, 2009). The relationships between reading ability and emotional problems do not appear to be mediated by ADHD or inattention (Arnold et al., 2005; Carroll et al., 2005) and it has been suggested that education-related stressors may play a crucial role (Maughan \& Carroll, 2006).

Whilst evidence for an association between reading ability and both externalising and internalising symptoms is accumulating, to date few studies have examined why reading difficulties might be associated with internalising and externalising symptoms. If we are to design effective interventions to promote mental health and reduce psychological distress for children with reading difficulties, it is essential to identify potentially malleable mechanisms explaining psychological outcomes for these children (Boyes et al., in press). A potential factor that warrants investigation is child self-esteem. Low self-esteem is a predictor of poor child mental health and 
Reading, self-esteem, and child mental health 5

psychological adjustment more generally (Allgood-Merton, Lewinsohn, \& Hops, 1990; Harter \& Whitesell, 1996; Mann, Hosman, Schaalma, \& de Vries, 2004; Sowislo \& Orth, 2013) and importantly can be modified through intervention (Haney \& Durlak, 1998; O'Dea \& Abraham, 2000).

\section{Reading and child self-esteem}

There are differing views as to whether self-esteem should be conceptualised as a unitary construct or instead conceptualised as comprising multiple dimensions. For example, Rosenberg (1979) emphasized that self-esteem is an overarching, general, or global construct. In contrast, Harter (1985) proposed both a global domain of self-esteem, as well as more specific dimensions of scholastic competence (child's perceived cognitive competence regarding schoolwork) social acceptance (e.g. knowing how to make friends), physical appearance (e.g. happy with looks, body, face, hair), and behavioural conduct (degree to which a child likes the way they behave). Similar hierarchical models have been proposed by Marsh and colleagues (Marsh, 1986; Marsh, Craven, \& Martin, 2006), incorporating a global sense of wellbeing as well as perceptions regarding self-esteem in specific domains such as academic, social, parent-home, or physical appearance (Marsh \& O'Mara, 2008). From a developmental perspective, it has been suggested that self-esteem may follow a progression from relatively undifferentiated global self-esteem to a more differentiated and hierarchical model of self-esteem as children develop (Battle, 1990). This is likely to have implications for the measurement of self-esteem across childhood and adolescence.

A number of studies have examined relationships between reading ability and global selfesteem, as well as self-esteem in specific domains (McArthur, Francis, Caruana, Boyes, \& Badcock, 2016). With regard to general self-esteem, the findings are mixed. A number of studies have reported poor general self-esteem, general self-concept, or general self-worth in children with reading difficulties (Alexander-Passe, 2006; Humphrey \& Mullins, 2002); however, other 
Reading, self-esteem, and child mental health 6 studies have reported no deficits in general self-esteem (Frederickson \& Jacobs, 2001; Terras et al., 2009). There are also mixed findings regarding relationships between reading ability and social (Alexander-Passe, 2006; Boetsch et al., 1996; Terras et al., 2009), parent-home (Alexander-Passe, 2006; Humphrey \& Mullins, 2002), and physical self-esteem (Boetsch et al., 1996; Humphrey \& Mullins, 2002; Terras et al., 2009). In contrast, findings regarding academic self-esteem are more equivocal with the majority of studies reporting that reading difficulties are associated with poorer academic self-esteem (Alexander-Passe, 2006; Boetsch et al., 1996; Casey, Levy, Brown, \& Brooks-Gunn, 1992; Frederickson \& Jacobs, 2001; Humphrey \& Mullins, 2002; Snowling, Muter, \& Carroll, 2007; Terras et al., 2009).

\section{The current study}

Whilst acknowledging that previous findings regarding poor reading and self-esteem are mixed, given the association between self-esteem and mental health more generally (Mann et al., 2004), it is plausible that self-esteem may play an important role in the association between reading ability and mental health (Terras et al., 2009). However, studies tend to only examine relationships between reading ability and either child mental health or self-esteem, rather than examining relationships between reading ability, self-esteem, and mental health concurrently. Therefore, the current study aimed to examine relationships between reading ability, self-esteem, and child mental health in a sample of Australian children. It was hypothesised that $i$ ) reading ability would be negatively associated with child mental health (both externalising and internalising symptoms), and $i$ ) self-esteem would moderate and/or mediate the relationship between reading ability and child mental health. To the best of our knowledge, no study to date has examined potential moderating or mediating effects of self-esteem on the relationships between reading ability and child mental health. Given this, the current study was exploratory in nature and the possibility of both moderation and mediation were investigated. 
Reading, self-esteem, and child mental health 7

\section{Method}

\section{Participants}

Children aged between 7 to 12 years $(n=117, M=9.09, S D=1.32,60 \%$ female) participated in this study. Participants were native English speakers with normal or corrected to normal vision and no impairments in hearing or attention. Participants were not selected on the basis of their reading ability. Recruitment sources included two existing Macquarie University databases in which parents had previously agreed to be contacted regarding their child's potential inclusion in future studies; the Reading Training Study (RTS) database and the Neuronauts Brain Science Club database. Contact was made via telephone, email or online newsletter, inviting participation in the study. Additional avenues of recruitment included flyers, posters, and advertisements on campus at Macquarie University; on community noticeboards, in newspapers, and on the Macquarie University website.

\section{Measures}

Reading ability was assessed using the Castles and Coltheart Test 2 (CC2; Castles et al., 2009). The CC2 is a 120-item standardised measure of single-word reading ability. The 120 items are divided into three categories of 40 words: regular, irregular, and non-words. Regular word reading assesses both lexical and non-lexical word access and example items include; "bed", "long", "mist". Irregular word reading assess the lexical reading route, or whole word retrieval and example items include; "good", "wolf", "work". Non-word reading assesses the non-lexical reading route and example items include; "norf", "gop", "hest" (Castles et al., 2009). Participants are asked to read single words of increasing difficulty and a discontinue rule is applied on the basis of word category: if five successive words in a given category are incorrectly pronounced, this category is discontinued. Administration continues until all categories have been discontinued or all items have been administered. A computerised version of the CC2 was 
Reading, self-esteem, and child mental health 8

employed in this study. The total number of correctly pronounced words within each category were converted to standardised $z$-scores using Australian normative data (Castles et al., 2009).

Child mental health was measured by self-report using the Strengths and Difficulties Questionnaire (SDQ; Goodman, 1997), which was administered verbally in small groups (n $\leq 4)$. The 25 items of the SDQ are equally divided into five subscales, emotional problems (e.g., I am often unhappy, depressed or tearful), conduct problems (e.g., I get very angry and often lose my temper), hyperactivity (e.g., I am restless, I cannot stay still for long), peer relationship problems (e.g., Other children or young people pick on me or bully me), and pro-social behaviour (e.g., I am kind to younger children). Each item is rated on a 3-point Likert scale ( 0 : not true, 1 : somewhat true, 2: certainly true). A total difficulties score (ranging between 0 and 40 ) is generated by summing all subscales except the prosocial subscale. An externalising symptoms score (ranging between 0 and 20) is generated by summing the conduct problems and hyperactivity subscales. An internalising symptoms score (ranging between 0 and 20) is generated by summing the emotional problems and peer problems subscales (Goodman, 1997). The child-report version of the SDQ was designed for use with children aged between 11 and 17; however, when administered verbally it has been validated for use with children as young as six (Curvis, McNulty, \& Qualter, 2014). The internal consistencies for the total difficulties $(\alpha=.73)$, externalising symptoms $(\alpha=.74)$, and internalising symptoms $(\alpha=.69)$ demonstrated adequate reliability in the current sample.

Self-esteem was measured using the Coopersmith Self-esteem Inventory (Coopersmith, 1967). The inventory consists of 58 items responded to on a dichotomous scale (like me or unlike $m e$ ). It is based on a multidimensional view of self-esteem and contains five subscales; general self-esteem (e.g., I am pretty sure of myself), social self-esteem (e.g., I am popular with children my own age), self-esteem at home, (e.g., I get upset easily at home), school/academic self-esteem (e.g., I am proud of my schoolwork), and a lie scale measuring participant honesty (e.g., I am 
Reading, self-esteem, and child mental health 9

never unhappy). In the current study items were scored such that a higher total score was indicative of better self-esteem.

The internal consistencies of the social $(\alpha=.61)$, home $(\alpha=.54)$, and school/academic $(\alpha$ $=.59)$ subscales were poor in the current sample. Given the poor reliability of the inventory in the current sample it was examined using principal components analysis. An examination of the scree plot and eigenvalues suggested between 6 and 22 components with no clear structure emerging in the current sample. Therefore, in order to generate a simpler and more reliable measure of selfesteem, an additional principal components analysis was conducted in which a single component was stipulated. The 12 items with component loadings greater than .50 on this single component were retained in an abbreviated measure of self-esteem (see Appendix I for the abbreviated measure and component loadings). This scale demonstrated excellent reliability $(\alpha=.82)$ and included items relating to general self-esteem, as well as self-esteem in the academic, social, and home domains. The abbreviated measure was used in all analyses reported in the current study.

Non-verbal IQ was measured using the block design and matrix reasoning subscales of the Wechsler Abbreviated Scale of Intelligence $-1^{\text {st }}$ Edition (WASI; Psychological Corporation, 1999). The WASI is a short assessment of intellectual functioning for use with participants between 6 and 89 years. The WASI non-verbal IQ scale is highly correlated with total non-verbal (performance) scores on the full Wechsler Intelligence Scale for Children $-3^{\text {rd }}$ Edition $(r=.76)$, and the block design $(r=.73)$ and matrix reasoning $(r=.83)$ subscales of the WASI are also highly correlated with the non-verbal subscale of the Kaufman Brief Intelligence Test (Hays, Reas, \& Shaw, 2002). Administration and scoring was conducted by trained psychology researchers following the standardised procedures described in the WASI manual.

Demographic characteristics, including child, gender and age, were obtained via parent report. With regard to age, parents provided children's actual date of birth as this was required to generate age standardised scores on the CC2 and the WASI. 


\section{Procedure}

Participants were recruited as a part of a broader study into cognition and reading. Ethical approval was obtained from Macquarie University ethics committee and informed consent was obtained from a parent or guardian. Additionally, children assented to participate in the study and were free to withdraw at any time. Children undertook a maximum of 4.5 hours of testing across an 8 hour day (8:30am to 4:30pm), with regular rest breaks between testing procedures. The day was structured as a holiday program organised around a space theme. Assessments were introduced with stories and structured as challenges, for which children received credit points that contributed to entertainment activities during the 'play' sessions across the day. Reading and nonverbal intelligence assessments were administered in individual sessions in silent and comfortable testing rooms. The SDQ and self-esteem scale were administered in small groups $(n \leq 4)$ with items read allowed by a trained researcher. In these small group sessions children were sat at individual desks and could not see other children's responses. To ensure confidentiality but allow data to be matched across tasks, children were given a unique identification number and this was recorded for all assessments.

\section{Analysis Strategy}

Analyses were conducted in three stages in SPSS 21. First, descriptive statistics for reading variables, self-esteem, child mental health, and non-verbal IQ and were calculated and we checked for outliers. Second, correlations between reading ability, self-esteem, mental health, and potential confounders (age, gender, and non-verbal IQ) were examined. Finally, multivariate relationships between reading ability, self-esteem, and mental health were tested in a series of hierarchical linear regressions, adjusting for potential confounders. In order to determine whether self-esteem moderated the relationship between reading ability and mental health, an interaction term was created and tested in the regression models. Significant interactions were probed using simple slopes analysis (Aiken \& West, 1991). 
Reading, self-esteem, and child mental health 11

\section{Results}

\section{Descriptive Statistics}

Descriptive statistics for the reading scores, non-verbal IQ scores, self-esteem scores, internalising and externalising symptoms, and total difficulties scores are summarised in Table 1. Rates of missing data ranged between 2-4\%. Data were missing completely at random [Little's test: $\left.\chi^{2}(5),=2.89 p=.716\right]$ and listwise exclusion was implemented. No outliers were identified and alpha was set at $p<.05$ for all analyses.

\section{(Table 1 approximately here)}

The three reading variables were highly correlated $(r=.67$ to .73$)$ and were summed to create a composite reading ability variable $(\alpha=.88)$. The two non-verbal IQ measures were also highly correlated $(r=.61)$ and were summed to create a composite non-verbal IQ variable. The composite reading ability and non-verbal IQ variables are used in all further analyses.

\section{Correlations between reading ability, self-esteem, mental health, and potential confounders}

Correlations between reading ability, self-esteem, internalising and externalising symptoms, total difficulties scores, and potential confounders (age, gender, and non-verbal IQ) are presented in Table 2. Reading ability was negatively correlated with internalising symptoms and total difficulties on the SDQ, but was not correlated with self-esteem. Unexpectedly, self-esteem was not correlated with internalising symptoms, externalising symptoms, or total difficulties on the SDQ. Gender was not associated with any of the measured variables; however, age was negatively associated with reading ability and internalising symptoms. Additionally, non-verbal IQ was negatively associated with reading ability. Therefore, age and non-verbal IQ were controlled for in all further analyses. 
(Table 2 approximately here)

\section{Multivariate relationships between reading ability, self-esteem, and mental health}

Three multivariate linear regressions were conducted following four steps. Age and non-verbal IQ were controlled for in the first step. Reading ability was entered in the second step. Selfesteem was entered in the third step. The interaction between reading ability and self-esteem was entered in the final step. As the current research aimed to examine potential moderating effects of self-esteem on the relationship between reading ability and child mental health, all predictor variables were mean centred. These analyses are summarised in Table 3.

\section{(Table 3 approximately here)}

Total difficulties: After adjusting for age and non-verbal IQ, there was a significant negative association between reading ability and total difficulty score (Step 2), which was maintained when self-esteem was entered into the model (Step 3). Self-esteem was not significantly associated with total difficulty score on the SDQ (Step 3); however, the interaction between reading ability and self-esteem was significant (Step 4). This model accounted for 17\% of the variance in total difficulty scores. Simple slopes tests (illustrated in Figure 1) revealed a significant negative relationship between reading ability and total difficulties at low levels ( $1 S D$ below the mean) of self-esteem $(b=-1.04, t=-3.29, p=.001)$ but not at high levels (1SD above the mean) of self-esteem $(b=.15, t=.52, p=.604)$.

Externalising symptoms: After adjusting for age and non-verbal IQ, reading ability was not significantly associated with externalising symptoms (Step 2). Self-esteem was not significantly associated externalising symptoms (Step 3); however, the interaction between reading ability and self-esteem was significant (Step 4). This model accounted for 9\% of the variance in externalising symptoms. Simple slopes tests (illustrated in Figure 1) revealed a 
significant negative relationship between reading ability and externalising symptoms at low levels ( $1 S D$ below the mean) of self-esteem $(b=-.41, t=-2.13, p=.036)$ but not at high levels (1SD above the mean) of self-esteem $(b=.23, t=1.40, p=.165)$.

Internalising symptoms: After adjusting for age and non-verbal IQ, there was a significant negative association between reading ability and internalising symptoms (Step 2). Self-esteem was not significantly associated with internalising symptoms (Step 3) and the interaction between reading ability and self-esteem was not significant (Step 4). This model accounted for $14 \%$ of the variance in internalising symptoms. Although the interaction was non-significant, the pattern was the same as that obtained for total difficulties score and externalising symptoms (illustrated in Figure 1).

\section{(Figure 1 approximately here)}

As self-esteem was not correlated with reading ability and also was not significantly associated with total difficulties, externalising symptoms, or internalising symptoms in the multivariate analyses, formal tests of mediation were not conducted.

\section{Discussion}

Reading ability is associated with mental health in children, with poor readers being at elevated risk of both internalising and externalising problems (Carroll et al., 2005; Maughan \& Carroll, 2006). However, almost no research has examined why reading ability is associated with these negative outcomes (Boyes et al., in press). Previous research has also established that reading difficulties are associated with poor academic self-esteem (Alexander-Passe, 2006; Boetsch et al., 1996; Casey et al., 1992; Frederickson \& Jacobs, 2001; McArthur et al., 2016), with some evidence of an additional deficit in global self-esteem and self-worth (Alexander-Passe, 2006; Humphrey \& Mullins, 2002). The current study aimed to investigate the possibility that the 
Reading, self-esteem, and child mental health 14 relationship between reading ability and child mental health may be moderated and/or mediated by self-esteem.

After adjusting for potential confounders (i.e., age and non-verbal IQ), reading ability was negatively associated with internalising symptoms and total difficulty scores on the SDQ. These findings are consistent with previous research demonstrating associations between reading ability and internalising symptoms (Arnold et al., 2005; Carroll et al., 2005). However, there was no association between reading ability and externalising symptoms. This was unexpected, as a large body of research has demonstrated that reading difficulties are associated with disruptive behaviour, and in particular inattention and hyperactivity (Arnold et al., 2005; Carroll et al., 2005; Frick et al., 1991; Willcutt \& Pennington, 2000b). One possible explanation for these findings is that the current sample was not selected based on reading disability, instead we examined associations between reading ability more generally and child mental health. Previous findings of comorbidity between poor reading and externalising behaviours have typically used reading disordered samples and these associations may only emerge in children with more severe reading difficulties (Arnold et al., 2005). An additional consideration is that mental health was measured using child self-report in the current study. Whilst this may be a strength of the study in terms of measuring internalising symptoms (as internal emotional states are likely to be less observable to parents; Verhulst \& van der Ende, 1992), children may be less accurate in selfdisclosing disruptive behavioural problems (Edelbrock, Costello, Dulcan, Conover, \& Kalas, 1986). Future research should examine relationships between reading ability, self-esteem, and mental health utilising both child and parent reports, as both the convergence and divergence of information obtained from children and parents is likely to be of interest.

However, self-esteem did interact with reading ability in predicting externalising symptoms and also moderated the relationship between reading ability and total difficulties scores on the SDQ. With regard to externalising symptoms, simple slopes analyses indicated that 
Reading, self-esteem, and child mental health 15 there was an association between poor reading ability and externalising symptoms, but only for children with low levels of self-esteem (Figure 1). The identical pattern of findings was obtained for total difficulties scores, and although it did not reach statistical significance, the same pattern was observed for internalising symptoms. Taken together, these findings indicate that self-esteem may be an important moderator of relationships between reading ability and child mental health and warrants further investigation. No evidence that self-esteem mediated the relationship between reading ability and child mental health was obtained. Unexpectedly, self-esteem was not significantly associated with any of the mental health outcomes (although the patterns were in the expected direction) or reading ability. This may have been due to the measurement of self-esteem in the current study. The internal consistencies of the Coopersmith Self-esteem Inventory subscales were poor in the current sample. This may have been due the young age of the sample, as it has been suggested that the development of self-esteem may follow a progression from relatively undifferentiated global self-esteem to a more differentiated and hierarchical model of self-esteem as children develop (Battle, 1990). Given the poor reliability of the subscales, an abbreviated self-esteem scale was created based on top loading items identified in a principal components analysis (with component loadings $>.50$ ). This abbreviated scale demonstrated excellent reliability; but did not allow for distinctions between global self-esteem and more specific domains of self-esteem. This is a limitation of the study, particularly given that academic self-esteem is most closely associated with reading ability in previous studies (Alexander-Passe, 2006; Boetsch et al., 1996; Casey et al., 1992; Frederickson \& Jacobs, 2001).

It should also be noted that the current study focused specifically on self-esteem as a potential moderator and/or mediator of the association between reading ability and child mental health. Reading ability is only one of many factors related to child mental health. Additionally, it is possible that children with reading difficulties may have experienced life circumstances or events which may have impacted on both their reading ability and mental health. This may be particularly relevant given that as the program was operating out of a university setting it is likely 
Reading, self-esteem, and child mental health 16 that participants were disproportionately from mid- to high socio-economic status backgrounds. Socio-ecological frameworks (Bronfrenbrenner, 1979) situate the child at the centre of interacting influences operating at different levels (e.g. individual, family, and community), and argue that resources in one area may buffer against deficits in another. While beyond the scope of the current study, further research investigating other potential risk (e.g. bullying victimisation, family instability) and protective (e.g. coping skills, emotion regulation) factors that might moderate and/or mediate associations between reading ability and child mental health would clearly be beneficial (Boyes et al., in press).

Bearing the limitations mentioned above in mind, the current study demonstrates that reading ability is associated with internalising symptoms and that self-esteem moderates the impact of reading ability on externalising symptoms and total difficulty scores. This is an important and novel finding, which suggests that children's self-esteem can help buffer against the negative mental health impacts of poor reading ability. However, given the limitations of the self-esteem measure, replication of these findings using a reliable and valid self-esteem measure, as well as other measures of child mental health, is clearly needed. From an intervention perspective, there is evidence that school-based programs, integrated into the curriculum, can improve children's self-esteem (O'Dea \& Abraham, 2000), as well as meta-analytic evidence that self-esteem can be improved (Haney \& Durlak, 1998). If the moderating effects of self-esteem on associations between reading ability and child mental health can be replicated then future research should examine whether interventions aiming to improve child self-esteem (either through universal curriculum-based programs or individualised programs) can reduce the risk of mental health problems in children with reading difficulties, particularly when these supplement ongoing evidence-based intervention to address the child's reading difficulties. Finally, given the direct association between reading ability and internalising symptoms, research examining whether universal school-based mental health promotion programs can improve the emotional health of children with reading difficulties may be beneficial. School-based prevention and 
Reading, self-esteem, and child mental health 17 intervention programs are cost-effective and have been shown to reduce risks for the development of emotional problems in school children (Neil \& Christensen, 2009). Given the majority of school children will not develop a mental health problem, it would be helpful to see if such programs are particularly effective for children with reading difficulties when administered alongside reading remediation programs. 
Reading, self-esteem, and child mental health 18

\section{Key Points}

\section{What is already known about this topic?}

1) Reading ability is associated with mental health, with poor readers at elevated risk of both externalising and internalising problems.

2) Reading ability is negatively associated with child self-esteem, although contradictory findings have been reported.

3) No research has examined whether self-esteem moderates and/or mediates relationships between reading ability and child mental health.

\section{What this paper adds?}

1) This paper demonstrates that self-esteem may moderate the relationship between reading ability and child mental health. Specifically, poor reading was only associated with externalising symptoms and total difficulties at low levels of self-esteem.

2) Poor reading ability was significantly associated with internalising symptoms, but this relationship was not moderated by self-esteem.

3) Interventions improving self-esteem may reduce the risk of mental health problems in children with reading difficulties, particularly if these supplement ongoing evidence-based intervention addressing reading difficulties.

\section{Acknowledgements:}

The authors would like to thank all volunteers who contributed to data collection. The research was supported by the following grants: Macquarie University Research Development Grants [9201200286, 9201200323], ARC Discovery Project Grant [DP0985138], Australian Research Council Centre of Excellence in Cognition and its Disorders [CE110001021], and a grant from the Research and Development Committee of the School of Psychology and Speech Pathology, Faculty of Health Sciences, Curtin University. 
Reading, self-esteem, and child mental health 19

\section{References}

Aiken, L. S., \& West, S. G. (1991). Multiple regression: Testing and interpreting interactions. Newbury Park: Sage.

Alexander-Passe, N. (2006). How dyslexic teenagers cope: An investigation of self-esteem, coping and depression. Dyslexia, 12, 26-275. doi:10.1002/dys.318

Allgood-Merton, B., Lewinsohn, P. M., \& Hops, H. (1990). Sex differences in adolescent depression. Journal of Abnormal Child Psychology, 99, 55-63. doi:10.1037/0021843X.99.1.55

Arnold, E., Goldston, D., Walsh, A., Reboussin, B., Daniel, S., Hickman, E., \& Wood, F. (2005). Severity of emotional and behavioral problems among poor and typical readers. Journal of Abnormal Child Psychology, 33, 205-217. doi:10.1007/s10802-005-1828-9

Battle, J. (1990). Self-esteem: The new revolution. Edmonton: James Battle Associates.

Boetsch, E. A., Green, P., \& Pennington, B. F. (1996). Psychosocial correlates of dyslexia across the life span. Development and Psychopathology, 8, 539-562. doi:10.1017/S0954579400007264

Boyes, M. E., Leitao, S., Claessen, M., Badcock, N. A., \& Nayton, M. (in press). Why are reading difficulties associated with mental health problems? Dyslexia.

Bronfrenbrenner, U. (1979). The ecology of human development: Experiments by nature and design. Cambridge: Harvard University Press.

Carroll, J. M., Maughan, B., Goodman, R., \& Meltzer, H. (2005). Literacy difficulties and psychiatric disorders: Evidence for comorbidity. Journal of Child Psychology and Psychiatry, 46, 524-532. doi:10.1111/j.1469-7610.2004.00366.x

Casey, R., Levy, S. E., Brown, K., \& Brooks-Gunn, J. (1992). Impaired emotional health in children with mild reading difficulty. Journal of Developmental and Behavioral Pediatrics, 13, 256-260. doi:10.1097/00004703-199208000-00003 
Reading, self-esteem, and child mental health 20

Castles, A., Colthart, M., Larsen, L., Jones, P., Saunders, S., \& McArthur, G. (2009). Assessing the basic components of reading: A revision of the Castles and Coltheart test with new norms. Australian Journal of Learning Difficulties, 14, 67-88. doi:10.1080/19404150902783435

Coopersmith, S. (1967). The antecedents of self-esteem. San Francisco: W. H. Freeman \& Co. Curvis, W., McNulty, S., \& Qualter, P. (2014). The validation of the self-report Strengths and Difficulties Questionnaire for use by 6- to 10-year-old children in the UK. British Journal of Clinical Psychology, 53, 131-137. doi:10.1111/bjc.12025

Edelbrock, C., Costello, A. J., Dulcan, M. K., Conover, N. C., \& Kalas, R. (1986). Parent-child agreement on child psychiatric symptoms assessed via structured interviews. Journal of Child Psychology and Psychiatry, 27, 181-190. doi:10.1111/j.14697610.1986.tb02329.x/pdf

Frederickson, N., \& Jacobs, S. (2001). Controllability attributions for academic performance and the perceived scholastic competence, global self-worth, and achievement of children with dyselxia. School Psychology International, 22, 401-416. doi:10.1177/0143034301224002

Frick, P. J., Kamphaus, R. W., Lahey, B. B., Loeber, R., Christ, M., Hart, E. L., \& Tannenbaum, L. (1991). Academic underachievement and the disruptive behavior disorders. Journal of Consulting and Clinical Psychology, 59, 289-294. doi:10.1037/0022-006X.59.2.289

Goodman, R. (1997). The Strengths and Difficulties Questionnaire: A research note. Journal of Child Psychology and Psychiatry, 38, 581-586. doi:10.1111/j.14697610.1997.tb01545.x/pdf

Haney, P., \& Durlak, J. A. (1998). Changing self-esteem in children and adolescents: A metaanalytical review. Journal of Clinical Child Psychology, 27, 423-433. doi:10.1207/s15374424jccp2704_6

Harter, S. (1985). The Self-Perception Profile for Children: Revision of the Perceived Competence Scale for Children. Denver: University of Denver. 
Reading, self-esteem, and child mental health 21

Harter, S., \& Whitesell, N. R. (1996). Multiple pathways to self-reported depression and psychological adjustment among adolescents. Development and Psychopathology, 8, 761777. doi:10.1017/S0954579400007410

Hays, J., Reas, D. L., \& Shaw, J. (2002). Concurrent validity of the Weschler Abbreviated Scale of Intelligence and the Kaufman Brief Intelligence Test among psychiatic inpatients. Psychological Reports, 90, 355-359. doi:10.2466/PR0.90.2.355-359

Hinshaw, S. P. (1992). Externalizing behavior problems and academic underachievement in childhood and adolescence: Causal relationships and underlying mechanisms. Psychological Bulletin, 111, 127-155. doi:10.1037/0033-2909.111.1.127

Humphrey, N., \& Mullins, P. M. (2002). Self-concept and self-esteem in developmental dyslexia. Journal of Research in Special Educational Needs, 2. doi:10.1111/j.14713802.2002.00163.x

Mann, M., Hosman, C., Schaalma, H., \& de Vries, N. (2004). Self-esteem in a broad-spectrum approach for mental health promotion. Health Education Research, 19, 357-372. doi:10.1093/her/cyg041

Marsh, H. W. (1986). Global self esteem: It's relation to specific facets of self-concept and their importance. Journal of Personality and Social Psychology, 51, 1224-1236. doi:10.1037/0022-3514.51.6.1224

Marsh, H. W., Craven, R., \& Martin, A. (2006). What is the nature of self-esteem? Unidimensional and multidimensional perspectives. In M. Kernis (Ed.), Self-esteem: Issues and answers (pp. 16-25). New York: Psychology Press.

Marsh, H. W., \& O'Mara, A. (2008). Reciprocal effects between academic self-concept, selfesteem, achievement, and attainment over seven adolescent years: Unidimensional and multidimensional perspectives of self-concept. Personality and Social Psychology Bulletin, 34, 542-552. doi:10.1177/0146167207312313 
Reading, self-esteem, and child mental health 22

Maughan, B., \& Carroll, J. M. (2006). Literacy and mental disorders. Current Opinion in Psychiatry, 19, 350-355. doi:10.1097/01.yco.0000228752.79990.41

McArthur, G. M., Francis, D., Caruana, N., Boyes, M. E., \& Badcock, N. A. (2016). Self-concept in poor readers: A systematic review protocol. PeerJ Preprints, 4, e2062v2061. doi:10.7287/peerj.preprints.2062v1

Neil, A., \& Christensen, H. (2009). Efficacy and effectiveness of school-based prevention and early intervention programs for anxiety. Clinical Psychology Review, 29, 208-215. doi:10.1016/j.cpr.2009.01.002

O'Dea, J., \& Abraham, S. (2000). Improving the body image, eating attitudes, and behaviors of young male and female adolescents: a new educational approach that focuses on selfesteem. International Journal of Eating Disorders, 28, 43-57. doi:10.1002/(SICI)1098108X(200007)28:1<43::AID-EAT6>3.0.CO;2-D

Psychological Corporation. (1999). Wechsler Abbreviated Scale of Intelligence (WASI). San Antonio: Harcourt Assessment.

Rietz, C. S., Hasselhorn, M., \& Labuhn, A. S. (2012). Are externalizing and internalizing difficulties of young children with spelling impairment related to their ADHD symptoms? Dyslexia, 18, 174-185. doi:10.1002/dys.1442

Rosenberg, M. (1979). Conceiving the self. New York: Basic Books.

Rutter, M., \& Yule, W. (1970). Reading retardation and antisocial behavior: The nature of the association. In M. Rutter, J. Tizard, \& K. Whitmore (Eds.), Education health and behavior (pp. 240-255). London: Longmans.

Snowling, M. J., Muter, V., \& Carroll, J. M. (2007). Children at family risk of dyslexia: a followup in early adolescence. Journal of Child Psychology and Psychiatry, 48, 609-618. doi:10.1111/j.1469-7610.2006.01725.x 
Reading, self-esteem, and child mental health 23

Sowislo, J. F., \& Orth, U. (2013). Does low self-esteem predict depression and anxiety? A metaanalysis of longitudinal studies. Psychological Bulletin, 139, 213-240.

doi:10.1037/a0028931

Terras, M., Thompson, L., \& Minnis, H. (2009). Dyslexia and psycho-social functioning: An exploratory study of the role of self-esteem and understanding. Dyslexia, 15, 304-327. doi:10.1002/dys.386

Verhulst, F. C., \& van der Ende, J. (1992). Agreement between parents' reports abd adolescents' self-reports of problem behavior. Journal of Child Psychology and Psychiatry, 33, 10111023. doi:10.1111/j.1469-7610.1992.tb00922.x

Whitehouse, A., Spector, T., \& Cherkas, L. (2009). No clear genetic influences on the association between dyslexia and anxiety in a population-based sample of female twins. Dyslexia, 15, 282-290. doi:10.1002/dys.378

Willcutt, E. G., \& Pennington, B. F. (2000a). Comorbidity of reading disability and attentiondeficit/hyperactivity disorder: Differences by gender and subtype. Journal of Learning Disabilities, 33, 179-191. doi:10.1177/002221940003300206

Willcutt, E. G., \& Pennington, B. F. (2000b). Psychiatric comorbidity in children and adolescents with reading disability. Journal of Child Psychology and Psychiatry, 41, 1039-1048. doi:10.1111/1469-7610.00691 
Table 1. Mean, standard deviation (SD), and range of scores for the reading, non-verbal IQ, selfesteem, and mental health variables

\section{Mean (SD) Range of scores $\quad$ Mean age-normed scores (SD)}

\section{Reading Variables}

Regular word reading

$33.20(6.49) \quad 13-40$

$.28(1.17)$

Irregular word reading

$20.93(6.06) \quad 5-33$

$.25(1.04)$

Non-word reading

$25.54(10.65) \quad 0-40$

$.01(1.07)$

Non-verbal IQ

Block design

$24.11(14.80) \quad 0-31$

$56.71(11.71)$

Matrices

$20.34(6.49) \quad 27-74$

$55.03(10.14)$

Self-Esteem

Abbreviated self-

$9.17(2.87) \quad 0-12$

esteem scale

$S D Q$ scores

Total difficulties

$17.33(5.50) \quad 8-34$

Externalising

$4.48(3.31) \quad 0-15$

symptoms

Internalising symptoms $4.64(3.28) \quad 0-17$

Note: Age-normed scores for the reading variables are $z$ scores $(M=0, S D=1)$. Age-normed scores for non-verbal IQ variables are $T$ scores $(M=50, S D=10)$. 
Table 2. Correlations between reading ability, self-esteem, mental health, and potential confounders (age, gender, and non-verbal IQ)

\begin{tabular}{|c|c|c|c|c|c|c|c|c|}
\hline & $\begin{array}{l}\text { Reading } \\
\text { ability }\end{array}$ & $\begin{array}{l}\text { Self- } \\
\text { esteem }\end{array}$ & $\begin{array}{l}\text { Internalising } \\
\text { symptoms }\end{array}$ & $\begin{array}{l}\text { Externalising } \\
\text { symptoms }\end{array}$ & $\begin{array}{l}\text { SDQ Total } \\
\text { Difficulties }\end{array}$ & Age & Gender & $\begin{array}{l}\text { Non-verbal } \\
\text { IQ }\end{array}$ \\
\hline Reading ability & -- & & & & & & & \\
\hline Self-esteem & .05 & -- & & & & & & \\
\hline $\begin{array}{l}\text { Internalising } \\
\text { symptoms }\end{array}$ & $-.19^{*}$ & -.16 & -- & & & & & \\
\hline $\begin{array}{l}\text { Externalising } \\
\text { symptoms }\end{array}$ & -.10 & -.15 & $.49^{* * *}$ & -- & & & & \\
\hline $\begin{array}{l}\text { SDQ Total } \\
\text { difficulties }\end{array}$ & $-.22 * *$ & -.16 & $.87 * * *$ & $.79 * * *$ & -- & & & \\
\hline Age & $-.19 *$ & .11 & $-.21^{*}$ & -.01 & -.13 & -- & & \\
\hline Gender & .07 & .09 & -.12 & .10 & -.06 & .10 & -- & \\
\hline Non-verbal IQ & $.41 * * *$ & -.01 & -.10 & -.09 & -.16 & -.05 & .00 & -- \\
\hline
\end{tabular}


Note: $* p<.05, * * p<.01, * * * p<.001$. With the exception of associations with gender, Pearson's $r$ is reported. As gender is a dichotomous variable Spearman's rho is reported for associations between gender and reading ability, self-esteem, mental health, age, and non-verbal IQ. 
Table 3. Summary of multivariate linear regressions for total difficulties, externalising symptoms, and internalising symptoms

\begin{tabular}{|c|c|c|c|c|c|c|c|c|c|c|c|c|}
\hline & \multicolumn{4}{|c|}{ Total difficulties } & \multicolumn{4}{|c|}{ Externalising symptoms } & \multicolumn{4}{|c|}{ Internalising symptoms } \\
\hline & $B(\mathrm{SE})$ & $\beta$ & $R^{2}$ & $\Delta R^{2}$ & $B$ & $\beta$ & $R^{2}$ & $\Delta R^{2}$ & $B$ & $\beta$ & $R^{2}$ & $\Delta R^{2}$ \\
\hline \multicolumn{13}{|l|}{ Step 1} \\
\hline Age & $-.60(.40)$ & -.14 & & & $-.03(.24)$ & -.01 & & & $-.55(.24)$ & $-.22 *$ & & \\
\hline Non-verbal IQ & $-.07(.03)$ & $-.20^{*}$ & .06 & $.06^{*}$ & $-.02(.02)$ & -.12 & .01 & .01 & $-.03(.02)$ & -.13 & .06 & $.06^{*}$ \\
\hline \multicolumn{13}{|l|}{ Step 2} \\
\hline Age & $-.78(.40)$ & -.19 & & & $-.06(.25)$ & -.03 & & & $-.66(.24)$ & $-.26 * *$ & & \\
\hline Non-verbal IQ & $-.04(.03)$ & -.11 & & & $-.02(.02)$ & -.09 & & & $-.01(.02)$ & -.04 & & \\
\hline Reading ability & $-.40(.20)$ & $-.22 *$ & .09 & $.03 *$ & $-.07(.12)$ & -.07 & .02 & .01 & $-.25(.12)$ & $-.23^{*}$ & .10 & $.04 *$ \\
\hline \multicolumn{13}{|l|}{ Step 3} \\
\hline Age & $-.71(.40)$ & -.17 & & & $-.01(.25)$ & -.01 & & & $-.62(.24)$ & $-.24 *$ & & \\
\hline Non-verbal IQ & $-.04(.03)$ & -.11 & & & $-.02(.02)$ & -.10 & & & $-.01(.02)$ & -.05 & & \\
\hline Reading ability & $-.38(.20)$ & $-.20 *$ & & & $-.06(.12)$ & -.05 & & & $-.24(.12)$ & $-.21 *$ & & \\
\hline Self-esteem & $-.27(.18)$ & -.14 & .11 & .02 & $-.17(.11)$ & -.15 & .04 & .02 & $-.15(.11)$ & -.13 & .12 & .02 \\
\hline
\end{tabular}


Reading, self-esteem, and child mental health 28

\begin{tabular}{|c|c|c|c|c|c|c|c|c|c|c|c|c|}
\hline \multicolumn{13}{|l|}{ Step 4} \\
\hline Age & $-.75(.39)$ & -.18 & & & $-.04(.25)$ & -.01 & & & $-.63(.24)$ & $-.25^{*}$ & & \\
\hline Non-verbal IQ & $-.03(.03)$ & -.08 & & & $-.01(.02)$ & -.07 & & & $-.01(.02)$ & -.03 & & \\
\hline Reading ability & $-.45(.19)$ & $-.24 *$ & & & $-.09(.12)$ & -.09 & & & $-.26(.12)$ & $-.23^{*}$ & & \\
\hline Self-esteem & $-.23(.18)$ & -.12 & & & $-.15(.11)$ & -.13 & & & $-.13(.11)$ & -.12 & & \\
\hline Reading*Self-esteem & $.20(.08)$ & $.25^{* *}$ & .17 & $.06^{* *}$ & $.11(.05)$ & $.22 *$ & .09 & $.05^{*}$ & $.07(.05)$ & .14 & .14 & .02 \\
\hline
\end{tabular}

Note: $* p<.05, * * p<.01$ 


\section{$\underline{\text { Figure Caption }}$}

Figure 1: Interactions between reading ability and self-esteem with regard to total difficulties, externalising, and internalising symptoms

1a Total difficulties

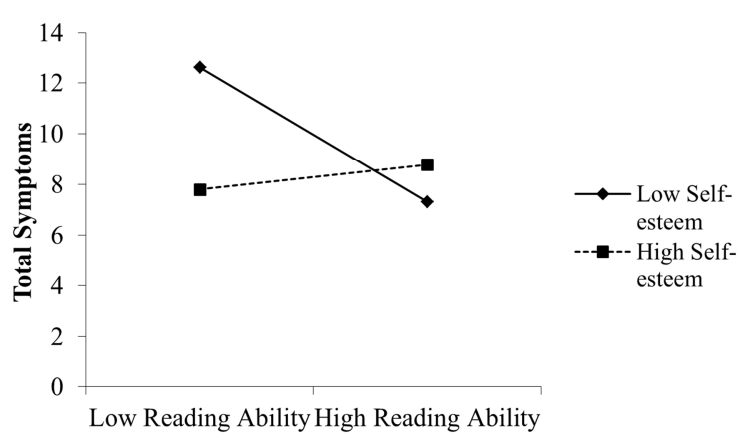

1b Externalising symptoms

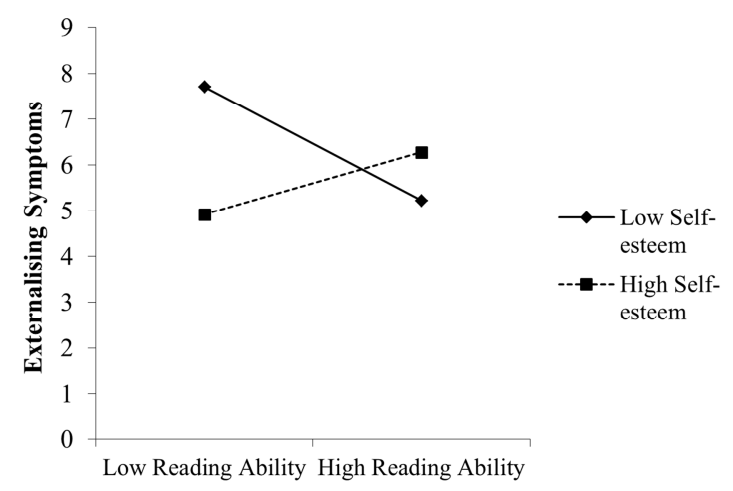

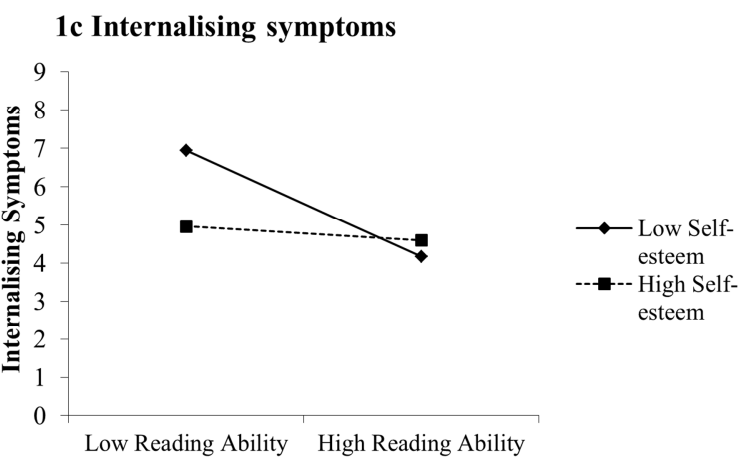


Appendix I (Supporting Information): Abbreviated Self-Esteem Scale

Item

Component loading

42. I often feel upset in school

48. My teacher makes me feel I am not good enough

50. I am a failure

58. I like most things about myself

30. It is pretty tough to be me

40. There are many times when I would like to leave home

7. I find it very hard to talk in front of the class

35. I am not doing as well in school as I would like to

31. Things are all mixed up in my life

15. Someone always has to tell me what to do

38. I have a low opinion of my self

45. Children pick on me very often

Note: Items are scored such that a higher score is indicative of better self-esteem 